\title{
Management of heart transplant recipients with hemodynamically significant clinical rejection in the presence of antibodies against angiotensin II type 1 receptor: A retrospective study
}

\author{
Puneet K Randhawa $^{1 \#}$, Mary Carmelle Philogene ${ }^{2 * *}$, Melissa Jeresano ${ }^{2}$, Marc K Halushka ${ }^{3}$ and Stuart Russell ${ }^{4}$ \\ ${ }^{1}$ Department of Medicine, MedStar Union Memorial Hospital, Baltimore MD \\ ${ }^{2}$ Department of Medicine, Johns Hopkins University School of Medicine, Baltimore,MD \\ ${ }^{3}$ Department of Pathology, Johns Hopkins School of Medicine, Baltimore, MD \\ ${ }^{4}$ Department of Medicine, Division of Cardiology, Duke University School of Medicine, Durham, NC

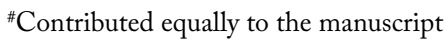

\begin{abstract}
Antibodies against Angiotensin II Type 1 Receptor (AT1R-Abs) have been associated with allograft rejection in heart transplantation. Data is lacking for modalities to mitigate their effect on cardiac function. We describe a case series consisting of patients with clinical and/or biopsy indication of rejection with detectable AT1R$\mathrm{Ab}$ and present treatment regimen and outcome. Clinical dysfunction was defined as hemodynamically significant cardiac dysfunction on echocardiogram including a decrease in ejection fraction greater than or equal to $20 \%$, shortness of breath, palpitations, chest tightness, and/or prolonged inotrope/vasopressor requirements during hospitalization. All patients selected for AT1R-Ab assessment had very low level or no donor specific HLA antibodies. Five of 9 patients with positive AT1R$\mathrm{Ab}$ recovered good graft function following treatment with either losartan alone or in combination with plasmapheresis and IVIG. Two patients did not recover normal graft function and died despite the use of similar treatments. Losartan and plasmapheresis are obvious therapeutic approaches for heart transplant recipients with clinical dysfunction and positive AT1R-Ab. Losartan blocks activation of AT1 receptor, and plasmapheresis reduces circulating antibodies. Additional considerations such as patient characteristics, comorbidities, the use of ARBs other than losartan and appropriate time for treatment administration need to be evaluated in larger studies.
\end{abstract}

\begin{abstract}
Abbreviations: AMR: antibody mediated rejection; ARB: angiotensin receptor blocker; AT1R: angiotensin II type 1 receptor; AT1R-Ab: anti-angiotensin II type 1 receptor antibody; ECHO: echocardiogram; EF: ejection fraction; HLA-DSA: donor specific HLA antibody; IVIG: intravenous Immunoglobulin; LHC: left heart catheterization; MFI: mean fluorescent intensity; NSVT: non sustained ventricular tachycardia; OHT: orthotopic heart transplantation; RHC: right heart catheterization
\end{abstract}

\section{Introduction}

Heart transplantation is a lifesaving treatment for patients with end stage heart failure. Despite improvements in medical procedures and immunosuppressive therapy, the 5 year patient survival after heart transplantation remains at $77 \%$ [1]. After surviving the immediate perioperative period, early complications facing the cardiac transplant population include infection, graft rejection, and cardiac allograft vasculopathy (CAV). Antibodies against the human leukocyte antigens (HLA) are known to play a dominant role in decreased graft survival [2] and treatments prior to and after transplantation are used for patients who present with HLA antibodies directed against donor antigens (HLA-DSA) [3]. Many centers also report testing for presence of antibodies other than HLA (non-HLA antibodies) when allograft dysfunction occurs in the absence of detectable HLA-DSA [4,5]; these include antibodies directed against the MHC class I polypeptiderelated sequence A [6], endothelin A receptor [7], vimentin and myosin
[8]. However, appropriate treatment approaches or outcomes posttreatment in the presence of non-HLA antibodies are not sufficiently described in the literature [3].

Antibodies against the $\mathrm{G}$ protein coupled receptor, angiotensin II type 1 receptor (AT1R-Abs) are non-HLA antibodies that have been implicated in heart allograft rejection. AT1R-Abs are associated with antibody-mediated rejection (AMR), cellular-mediated rejection (CMR), and early onset of microvasculopathy at 1 year posttransplantation [7]. Angiotensin II type 1 receptor (AT1R), the target for antibody binding, is expressed on the vascular endothelium and expression increases with inflammation caused by stress or graft injury [9]. AT1R mRNA expression was upregulated on the biopsy of heart transplant recipients with recurrent acute rejection [10]. As with other non-HLA antibodies, despite numerous reports on the involvement of AT1R-Ab in heart transplantation outcome, there are very few studies that focus on a treatment protocol in the presence of circulating

${ }^{\star}$ Correspondence to: Mary Carmelle Philogene, Immunogenetics Laboratory, 2041 E. Monument Street, Baltimore, MD 21205, USA, Tel: 410-955-3600; Fax: 410-955-0431; E-mail: mphilogene@jhmi.edu

Key words: non-HLA antibodies, angiotensin II type 1 receptor antibody, heart allograft dysfunction

Received: November 02, 2018; Accepted: November 13, 2018; Published: November 16, 2018 
Randhawa PK (2018) Management of heart transplant recipients with hemodynamically significant clinical rejection in the presence of antibodies against angiotensin II type 1 receptor: A retrospective study

AT1R-Ab. Here, we present a case series of heart transplant recipients with positive AT1R-Ab and decreased cardiac function and discuss therapeutic approaches used and outcome.

\section{Methods}

\section{Definition of "decreased cardiac function"}

This study was approved by the Johns Hopkins Hospital Institutional Review Board. Cardiac transplant patients at Johns Hopkins were routinely monitored for signs of rejection after transplantation with echocardiograms and endomyocardial biopsies. For patients between biopsies or who presented with signs or symptoms of heart failure, an echocardiogram was performed followed by a right heart catheterization and endomyocardial biopsy, if clinically indicated. For the purpose of this study, we defined hemodynamically significant cardiac dysfunction on echocardiogram as a decrease in ejection fraction (EF) of greater than or equal to $20 \%$. Symptoms including shortness of breath, palpitations, chest tightness, and prolonged inotrope/vasopressor requirements during hospitalization were other triggers for evaluation of cardiac function with echocardiogram with or without right heart catheterization (RHC) and cardiac biopsy. Biopsy proven AMR, decreased ejection fraction, or signs of hemodynamic insufficiency triggered testing for HLA-DSA. If HLA-DSA was negative or detected at very low levels that did not correlate with observed dysfunction $[3,11]$ testing for AT1R-Ab was performed.

\section{Biopsy evaluations}

Cardiac biopsies were performed per protocol during the first year post transplantation, at 1 to 4 weeks and months $2,3,4,5,6,8,10$ and 12. After one year post transplantation, biopsies were performed only in cases of suspected allograft dysfunction (for-cause) or if patients had rejection during the first year. Right heart catheterizations were performed with each biopsy. Biopsies were graded for cellular and antibody mediated rejections based on ISHLT 2005 criteria [12]. AMR was defined as positive immunofluorescence staining for peri-capillary deposition of complement split products C4d and C3d.

\section{Pre-transplant and post-transplant treatment}

Induction therapy with basiliximab $(2 \mathrm{mg} / \mathrm{kg})$ was given on postoperative days 1 and 4 for patients deemed at higher risk for rejection or for those who required delay of calcineurin inhibitor therapy due to renal dysfunction. This included patients with a history of sensitization, young females, those with diabetes, baseline renal insufficiency, or pretransplant use of left ventricular assist devices. Treatment for AMR varied by patient but routinely included plasmapheresis and IVIG plus increasing routine immunosuppression. Some patients with positive AT1R-Ab were started on the angiotensin receptor blocker, losartan (25-100 mg as tolerated by patient), when permitted by clinical condition. Limiting factors for use of ARBs included hyperkalemia, renal dysfunction or hypotension. Patients were also treated with steroids and angiotensin converting enzymes inhibitor (ACEi) as needed or when ARB could not be used.

\section{HLA typing and antibody determination}

Heart transplant candidates and their cadaveric donors were typed for HLA-A, B, C, DR, DQ and DP by reverse sequence specific oligonucleotide assay (LABType ${ }^{\circledR}$, One Lambda Canoga Park, CA). HLA antibody testing was performed with pre and post-transplant patients' sera using the Luminex ${ }^{\text {mm }}$ pooled HLA antigen (LMX), the phenotype bead assay (LMID) (Immucor-Lifecodes, Stamford, CT) and a single antigen panel (One Lambda, Canoga Park, CA). HLA antibody levels were assigned as previously described [13]. Briefly, cytotoxic crossmatch positive (CDCXM + ), flow cytometric crossmatch positive (FCXM+), and FCXM- Luminex+ levels were reported for MFI values $>10,000,4,000$ to $<10,000$, and 2,000 to $<4000 \mathrm{MFI}$, respectively for HLA-A, HLA-B, and HLA-DR, and $>20,000,12,000$ and 4,000 for HLA-C, HLA-DQ and HLA-DP respectively. MFI below these values were reported as low level and those that lacked specificity patterns were reported as negative. Cytotoxicity crossmatches were performed prospectively when heart transplant candidates were sensitized against HLA antibodies or retrospectively for non-sensitized patients. Cytotoxic crossmatch tests were done with positively selected $\mathrm{T}$ and B lymphocyte targets as previously described [14]. Flow cytometric crossmatches were performed as previously described [15] when a donor specific HLA antibody detected on the Luminex panel was $>3000$ MFI. Hypotonic dialysis was performed to remove IgM autoantibodies and $\operatorname{IgG}$ immune complexes from sera and sera for crossmatch testing were back-dialyzed to achieve isotonicity.

AT1R-Ab testing was performed using quantitative ELISA (One Lambda, ThermoFisher) as previously described [16] using sera collected at time of graft dysfunction. If available, a pre-transplant serum was tested retrospectively. AT1R-Ab concentrations $\geq 17$ units/ $\mathrm{ml}$ were reported as positive.

\section{Results}

\section{Clinical characteristics of patients selected for AT1R-Ab evaluation}

Testing for AT1R-Ab was performed between July 2014 and July 2017 for 12 heart transplant recipients. Nine of the twelve patients (75\%) were positive for AT1R-Ab. The characteristics of patients and donors are listed in Table 1. The mean time from transplantation to development of cardiac dysfunction was 61 months (range 5 to 252 months). The majority of the patients were male (75\%) and were diagnosed with non-ischemic cardiomyopathies (92\%) prior to heart transplantation. Seven of the 12 patients were treated with a ventricular assisted device prior to transplantation. One patient received a kidney transplant prior to heart transplantation.

The twelve patients presented with various abnormal clinical features including development of palpitations secondary to

Table 1. Characteristics of 12 Heart Transplant Recipients Evaluated for AT1R-Ab

\begin{tabular}{|l|l|}
\hline Patient Characteristics & $61(5-252)$ \\
\hline Median months from transplant (range) & $41(25-67)$ \\
\hline Mean age at transplantation (range) & $6(50)$ \\
\hline Caucasian, n (\%) & $9(75)$ \\
\hline Male, n (\%) & $7(58)$ \\
\hline Use of LVAD (\%) & $1(8)$ \\
\hline Prior history of transplants n (\%) & \\
\hline${\text { Causes of cardiovascular disease leading to } \text { OHT }^{3}}^{3}$ & $1(8)$ \\
\hline ICM $^{1}$ & $11(92)$ \\
\hline NICM $^{2}$ & \\
\hline Donor Characteristics & $42(25-69)$ \\
\hline Mean age at transplantation (range) & $7(58)$ \\
\hline Caucasian, n (\%) & $9(75)$ \\
\hline Male n, (\%) & $4.5 / 6$ \\
\hline HLA class I mismatch (A,B,C) & $2.8 / 4$ \\
\hline HLA class II mismatch (DR,DQ) & \\
\hline
\end{tabular}

ICM: ischaemic cardiomyopathy

${ }^{2} \mathrm{NICM}$ : non-ischaemic cardiomyopathy

${ }^{3} \mathrm{OHT}$ : orthotopic heart transplant 
Randhawa PK (2018) Management of heart transplant recipients with hemodynamically significant clinical rejection in the presence of antibodies against angiotensin II type 1 receptor: A retrospective study

supraventricular tachycardia $(\mathrm{n}=1)$, pacemaker alert for non-sustained ventricular tachycardia $(\mathrm{n}=1)$, prolonged inotrope/vasopressor dependence on inpatient admission $(n=2)$, volume overload presenting as peripheral edema $(n=1)$, worsening shortness of breath $(n=4)$, decreased EF on routine surveillance $(n=7)$. Measurements on right heart catheterization (RHC) showed a cardiac index $<2.5$ in 10 of the 12 patients. Furthermore, 7 of 12 patients had a left heart catheterization (LHC) done at the time of AT1R-Ab evaluation, all of which showed no coronary disease significant enough to explain the cardiac dysfunction.

\section{HLA antibody and Biopsy characteristics}

Pre-transplant HLA antibody data was available for 8 of the 12 patients; the remaining 4 patients were transplanted at other institutions prior to being followed at Johns Hopkins, therefore had no available pre-transplant serum (Table 2). Of those transplanted at Johns Hopkins, 7 patients were negative and 1 was positive for HLA antibody prior to transplantation; this patient had HLA-DSA against A2, below a level sufficient to cause a positive crossmatch. All CDCXMs were negative at time of transplantation. Six patients were positive for AT1R-Ab prior to transplantation.

At time of cardiovascular dysfunction, 8 of the 12 patients remained negative for HLA antibodies. Six of the 8 patients were positive for AT1R-Ab. Four patients had detectable HLA-DSA directed against HLA class II; one patient developed both class I and class II HLA-DSA (Table 2). In all cases, the level of HLA-DSA was low and not sufficient to yield positive crossmatches [11]. Of the 4 patients with low level HLA-DSA, 3 were also positive for AT1R-Ab.

Biopsy data are shown in Table 2. At time of dysfunction, 3 of the 9 recipients with positive AT1R-Ab developed biopsy proven antibody mediated rejection; 3 had mild rejection (grade $1 \mathrm{R} / 1 \mathrm{~A}$ ), 2 had no rejection and 1 patient had no rejection but progressive fibrosis indicated on biopsy. The remaining 3 patients with AT1R-Ab negative had mild $(1 \mathrm{R} / 1 \mathrm{~A})$ rejection $(\mathrm{n}=2)$ or no rejection $(\mathrm{n}=1)$.

\section{Treatment and outcome}

The course of treatment and outcome for the nine patients with positive AT1R-Ab at time of cardiac dysfunction are illustrated in Figure 1. Follow-up testing for AT1R-Ab were performed for 8 of the 9 patients using sequential sera as treatment was administered and until resolution of the dysfunction. Patients 1 through 5 were treated with losartan at clinically tolerated doses. Three of the 5 patients $(1,2$ and 3) recovered good graft function and ejection fractions improved to $>55 \%$. Patient \#2 was additionally treated with plasmapheresis and IVIG. These patients maintained good graft function until the end of follow-up (patient 1:13.5 months; patient 2: 2 months and patient 3: 6 months after initiation of treatment (Figure 1). Ejection fraction did not improve for patients 4 and 5 despite use of losartan and both patients died from complications related to graft dysfunction (patient 4 at 11 months after start of treatment and patient 5 at 14 months after the start of treatment). Importantly, prior to progression to graft dysfunction, patient 4 had a heart block that required a pacemaker and patient 5 had multiple infections.

Three patients (patients 6,7 and 8) who also presented with positive AT1R-Ab and low ejection fraction were not given an ARB. Patient \#6 was not able to be treated with an ARB due to hyperkalemia. He developed fibrosis on biopsy, was treated with pulse steroids and recovered good allograft function until the end of follow-up (2.3 months). Patients 7 and 8 developed AMR on several biopsies in the absence of HLA-DSA. Both patients were treated with several rounds of plasmapheresis and IVIG. AT1R-Ab levels decreased and both patients regained good allograft function up to 1 month and 11 months respectively after initiation of treatment. Patient \#9 showed mild rejection on several biopsies, but maintained normal ejection fraction with no other clinical abnormalities. This patient was given an angiotensin converting enzyme inhibitor (ACEi) and maintained good graft function in the presence of high level of AT1R-Ab. Patient \#11 had weakly positive AT1R-Ab as well as low level class I and class II HLA-DSA at time of cardiac dysfunction with decreased ejection

Table 2. Antibody and Biopsy Data for Patients Evaluated for AT1R-Ab

\begin{tabular}{|c|c|c|c|c|c|c|c|c|c|c|c|c|c|}
\hline Patient & Race & Sex & Age $^{1}$ & $\begin{array}{l}\text { Patient } \\
\text { Status }\end{array}$ & $\begin{array}{l}\text { Post Tx }{ }^{2} \\
\text { (Months) }\end{array}$ & $\mathbf{E F}^{3}$ & $\begin{array}{l}\text { Pre Tx } \\
\text { HLA- } \\
\text { DSA }\end{array}$ & $\begin{array}{l}\text { Post Tx HLA- } \\
\text { DSA }^{4}\end{array}$ & $\begin{array}{l}\text { Pre Tx } \\
\text { AT1R- } \\
\text { Ab }\end{array}$ & $\begin{array}{l}\text { Post Tx } \\
\text { AT1R- } \\
\text { Ab }\end{array}$ & Biopsy diagnosis & Losartan & $\begin{array}{l}\text { Other } \\
\text { treatments }\end{array}$ \\
\hline 1 & AA & $\mathrm{F}$ & 43 & living & 224 & 45 & $\mathrm{U}$ & DQ7 & $\mathrm{U}$ & 25.36 & No rejection & Yes & No \\
\hline 2 & $\mathrm{C}$ & M & 25 & living & 252 & 30 & $\mathrm{U}$ & DR51 & $\mathrm{U}$ & $>40$ & Mild acute rejection 1R; AMR 1R & Yes & PP/IVIG \\
\hline 3 & $\mathrm{C}$ & M & 67 & living & 36 & 35 & $\mathrm{~N}$ & $\mathrm{~N}$ & $>40$ & 22.25 & Mild acute rejection $1 \mathrm{R}$ & Yes & Pulse steroids \\
\hline 4 & $\mathrm{C}$ & M & 56 & deceased & 98 & 45 & $\mathrm{~N}$ & $\mathrm{~N}$ & $\mathrm{U}$ & $>40$ & No rejection & Yes & Pulse steroids \\
\hline 5 & AA & M & 39 & deceased & 75 & 40 & $\mathrm{~N}$ & DR9, DQ8, DQ9 & $>40$ & $>40$ & AMR 1R & Yes & PP/IVIG \\
\hline 6 & $\mathrm{C}$ & M & 60 & living & 47 & 35 & $\mathrm{~N}$ & $\mathrm{~N}$ & $>40$ & $>40$ & No rejection/Progressive fibrosis & No & Pulse steroids \\
\hline 7 & AA & M & 35 & living & 5 & 65 & $\mathrm{~A} 2 *$ & $\mathrm{~N}$ & $>40$ & $>40$ & Moderate rejection 2R; AMR 1 & No & PP/IVIG \\
\hline 8 & AA & M & 51 & living & 13 & $>70$ & $\mathrm{~N}$ & $\mathrm{~N}$ & $>40$ & $>40$ & AMR1 & No & PP/IVIG \\
\hline 9 & AA & M & 37 & living & 39 & 55 & $\mathrm{~N}$ & $\mathrm{~N}$ & $>40$ & $>40$ & Mild rejection $1 \mathrm{R}$ & No & No \\
\hline 10 & $\mathrm{C}$ & M & 43 & living & 322 & 35 & $\mathrm{U}$ & $\mathrm{N}$ & $\mathrm{U}$ & 3.8 & No rejection & Yes & No \\
\hline 11 & $\mathrm{O}$ & $\mathrm{F}$ & 26 & $\begin{array}{l}\text { living/ } \\
\text { declining } \\
\text { graft } \\
\text { function }\end{array}$ & 33 & 50 & $\mathrm{U}$ & B18, B60, DQ7 & $\mathrm{U}$ & 13.38 & Mild rejection $1 \mathrm{R}$ & No & No \\
\hline 12 & $\mathrm{C}$ & $\mathrm{F}$ & 34 & living & 93 & 65 & $\mathrm{~N}$ & $\mathrm{~N}$ & $\mathrm{U}$ & 6.9 & Mild rejection 1R & No & $\begin{array}{l}\text { Bolus and } \\
\text { taper steroids }\end{array}$ \\
\hline
\end{tabular}

AA: African American; C: Caucasian; O: Asian; F: female; M: male; U: data unavailable; N: negative.

${ }^{1}$ Age: age at time of transplantation

${ }^{2}$ Tx: Transplant

${ }^{3} \mathrm{EF}$ : ejection fraction at time of AT1R-Ab evaluation

*A2 HLA-DSA detected prior to transplantation was below a level sufficient to yield a positive CDCXM or FCXM

$>40$ : AT1R-Ab concentrations were beyond the maximum level of detection of the assay

${ }^{4}$ Post Tx HLA-DSA: Single and cumulative HLA-DSA were FCXM- Lum+ levels 


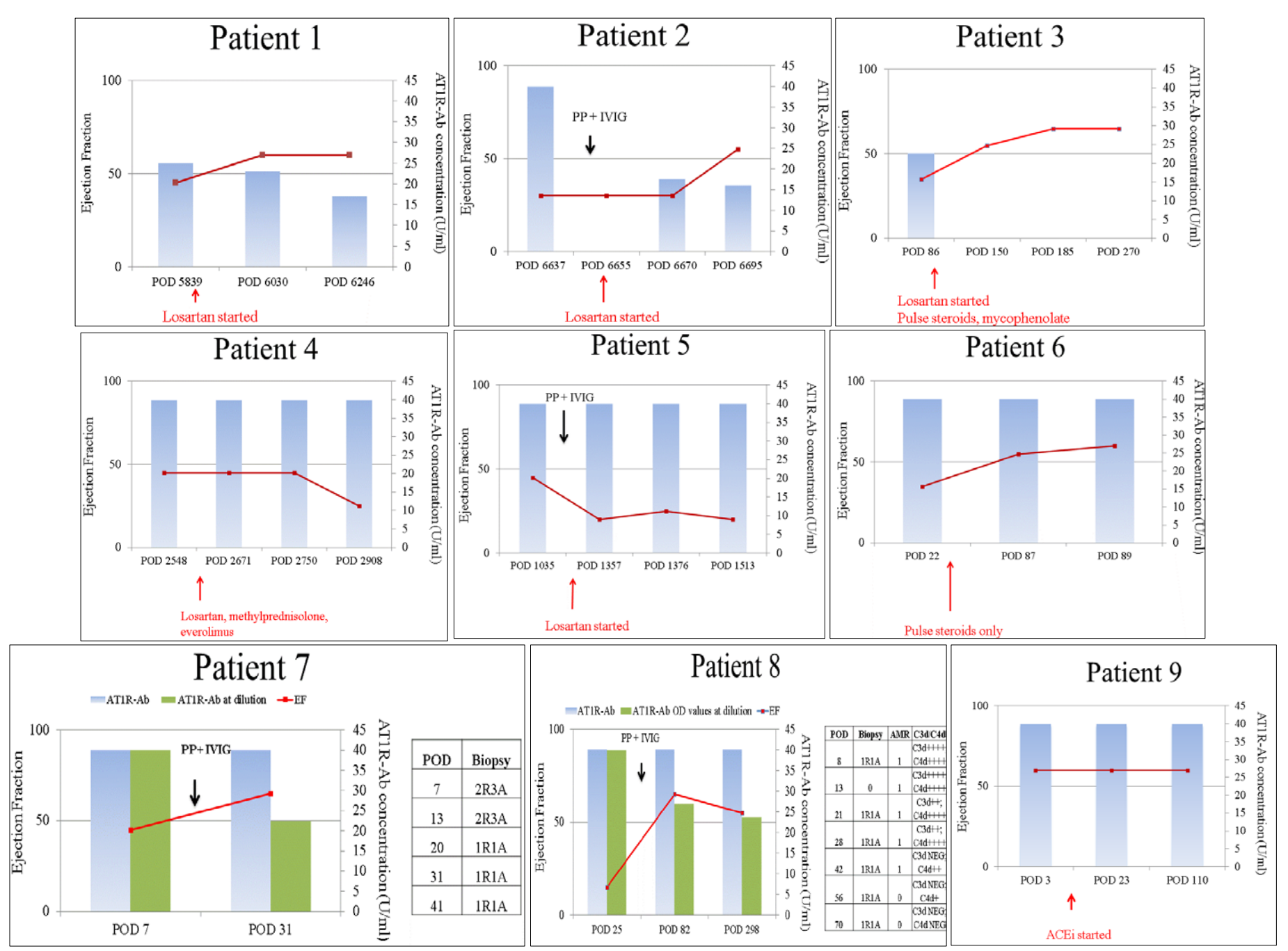

Figure 1. Treatment Protocol for 9 patients with positive AT1R-Ab. Heart transplant patients with graft dysfunction were treated with losartan, PP+IVIG, steroids, or ACEi. Percent ejection fraction (y axis - red line) and AT1R-Ab (U/ml) (x-axis- blue bars) were monitored overtime. Ejection fractions increased after treatment above $60 \%$ for patients $1,2,3,6,7,8$ (red lines). Ejection fraction remained below $60 \%$ for patients 4 and 5 . There was no change in ejection fraction for patient 9 . AT1R-Ab levels decreased for patients treated with plasmapheresis and IVIG $(1,2,3,7,8)$. POD $=$ Post-operative day.

fraction and mild cellular rejection (1R) on a biopsy. This patient never recovered good graft function and EF remained low until the end of follow up. Patient \#12 had mild rejection (1R) on biopsy with negative HLA-DSA and AT1R-Ab; this patient received steroids for the rejection (Table 2).

\section{Discussion}

In this study we describe the clinical course and treatment for heart transplant recipients who present with allograft dysfunction and positive AT1R-Ab. Of 12 patients evaluated, 9 were positive for AT1R$\mathrm{Ab}$. Seven patients recovered good graft function defined as resolution of clinical dysfunction as well as rejection on biopsy, and increase in ejection fraction. Five of the 7 patients received treatments consisting of either losartan alone, losartan with plasmapheresis and IVIG, or only plasmapheresis and IVIG. One patient (\#6) was given pulse steroids and remained stable with good graft function at 2 months after initiation of treatment with biopsy showing fibrosis. The final patient (\#9) was treated with an ACEi.

AT1R-Abs were shown to exert their effect by activating the G protein coupled receptor, AT1R, and initiating a pro-inflammatory cascade leading to endothelial damage. AT1R-Abs isolated from the sera of transplant recipients with vascular rejection induced Erk signaling in endothelial and vascular smooth muscle cells, resulting in the increased expression of inflammatory (MCP-1 and RANTES) and coagulation (Tissue Factor) proteins [17-19], reactive oxygen species, NFKB and NADPH oxidase expression [20]. Angiotensin receptors blockers, such as losartan, are small molecules with anti-inflammatory properties [21] that bind accessible epitopes of AT1R and function as inverse agonists [22-24]. Losartan is commonly used for treatment of cardiovascular diseases. However, two patients with positive AT1R-Ab never recovered normal graft function despite use of losartan. Several factors may account for these differences. First, AT1R activation via antibody binding rather than with the natural ligand, angiotensin II, could maintain the receptor in a constitutively active state. Zhang, et al. showed that vasoconstriction induced by AT1R-Ab was maintained for more than 30 minutes compared to 3 minutes with angiotensin II [25]. Studies that compare a wild type (stable) AT1R to an activated mutant AT1R show that interaction between AT1R residues and losartan may be altered when the receptor is maintained in an activated state [24]. The study further shows that other ARBs retain better inverse agonistic properties compared to losartan [24]. Therefore future 
Randhawa PK (2018) Management of heart transplant recipients with hemodynamically significant clinical rejection in the presence of antibodies against angiotensin II type 1 receptor: A retrospective study

studies comparing the efficacy of various ARBs may be needed to improve treatment protocols. Candesartan along with plasmapheresis was proposed as dual treatment in kidney transplant recipients who present with positive AT1R-Ab prior to transplantation [26]. Secondly, compared to the 7 patients who recovered good function, the 2 deceased patients had significant comorbidities; patient \#4 developed a heart block requiring a pacemaker prior to graft dysfunction and patient \#5 had several infections that were never resolved. Successful treatment may require initiation of therapeutic protocols in patients before they develop significant comorbidities.

AT1R-Ab was detected in the sera of 6 heart transplant recipients prior to transplantation (Table 2). In renal transplantation, patients with pre-transplant positive AT1R-Ab had increases in serum creatinine within month 3 post-transplantation and developed rejection [13]. Heart transplant recipients maintained on mechanical assist devices as a bridge to transplantation develop AT1R-Ab [27] and have a higher incidence of ischemic cardiomyopathy compared to those who did not use a ventricular assisted device (VAD) [28]. Biomaterials used in VAD were found to trigger host immune responses and lead to development of inflammation, fibrosis, coagulation, and infection [29]. Consequently, screening this category of patients for AT1R-Ab at time of transplantation and initiating treatment, may prevent early injury to the transplanted organ.

Plasmapheresis and IVIG have been used as desensitization treatments to reduce HLA antibody levels prior to transplantation or post-transplantation to treat AMR [3]. This desensitization treatment also reduces AT1R-Ab levels [30] although AT1R-Ab rebound often occurs after plasmapheresis treatments. Rapid removal of AT1R$\mathrm{Ab}$ at time of inflammation, particularly in patients with very high antibody titers, in conjunction with use of an appropriate ARB to block receptor activation was shown in patients 2,7 and 8 to be an effective combination of treatment.

One patient (patient \#6) was not treated with an ARB due to hyperkalemia but recovered good graft function following use of pulse steroid. A similar treatment was reported in a case study for treatment of rejection in the presence of AT1R-Ab [31]. AT1R expression increases during inflammation [9], therefore use of steroids to reduce inflammation may be an effective approach to reduce the availability of the target AT1R on the endothelium to which circulating antibodies can bind.

Four patients evaluated for AT1R-Ab also had HLA-DSA (patients $1,2,5,11$ ); the majority directed against HLA-DR and DQ antigens. Several studies in kidney transplantation have shown worst graft outcome when both HLA and AT1R antibodies are present in patient serum $[16,32]$. Additionally, a few studies have suggested that presence of AT1R-Ab prior to transplantation can elicit development of HLA antibodies [32].

This study has many limitations. As this was a retrospective analysis designed to provide some guidelines for treatment of patients with positive AT1R-Abs, there was no consistent treatment algorithm applied for all of the patients, and there was limited information available for the selection of one therapy versus another. Nevertheless, this small case series provides preliminary data for the design of larger, prospective studies that compare these treatments across larger populations and inform on best practices for patients with positive AT1R-Ab. There are certainly not enough patients in this study to infer any therapeutic benefit with treatment using losartan; however, since ARBs are commonly used to treat hypertension and cardiovascular diseases, clinicians are familiar with contraindications and appropriate doses. Further work is needed however to evaluate the efficacy of losartan versus other ARBs as well as best time to initiate treatment with an ARB.

AT1R-Ab in heart transplant recipients appears to be associated with hemodynamic significant rejection. The use of angiotensin receptor blockade can be considered in the therapy of these patients, in the presence of reduced ejection fraction, but further studies should be performed. In the setting of heart transplant patients with graft injury, evaluating for non-HLA antibodies should be considered.

\section{Disclosure statement}

The authors have no conflicts of interest to disclose.

\section{References}

1. Colvin M, Smith JM, Skeans MA (2017) OPTN/SRTR 2015 Annual Data Report: Heart. Am J Transplant 1: 286-356. [Crossref]

2. Campbell P (2013) Clinical relevance of human leukocyte antigen antibodies in liver, heart, lung and intestine transplantation. Curr Opin Organ Transplant 4: 463-469.

3. Kobashigawa J, Colvin M, Potena L, Dragun D, Crespo-Leiro MG, et al. (2018) The management of antibodies in heart transplantation: An ISHLT consensus document. $J$ Heart Lung Transplant.

4. Gates KV, Pereira NL, Griffiths LG (2017) Cardiac Non-Human Leukocyte Antigen Identification: Techniques and Troubles. Front Immunol 8: 1332

5. Kobashigawa J, Crespo-Leiro MG, Ensminger SM, Reichenspurner H, Angelini A, et al. (2011) Report from a consensus conference on antibody-mediated rejection in heart transplantation. J Heart Lung Transplant 30: 252-269.

6. Askar M, Hsich E, Reville P, Nowacki AS, Baldwin W, et al. (2013) HLA and MICA allosensitization patterns among patients supported by ventricular assist devices. $J$ Heart Lung Transplant 32: 1241-1248.

7. Hiemann NE, Meyer R, Wellnhofer E, Schoenemann C, Heidecke H, et al. (2012) Non-HLA antibodies targeting vascular receptors enhance alloimmune response and microvasculopathy after heart transplantation. Transplantation 94: 919-924.

8. Sharma M, Liu W, Perincheri S, Gunasekaran M, Mohanakumar T (2018) Exosomes expressing the self-antigens myosin and vimentin play an important role in syngeneic cardiac transplant rejection induced by antibodies to cardiac myosin. Am J Transplant.

9. Wassmann S, Stumpf M, Strehlow K, Schmid A, Schieffer B, et al. (2004) Interleukin-6 induces oxidative stress and endothelial dysfunction by overexpression of the angiotensin II type 1 receptor. Circ Res 94: 534-541.

10. Yamani MH, Cook DJ, Rodriguez ER, Thomas DM, Gupta S, et al. (2006) Increased expression of angiotensin II type 1 receptor (AGTR1) in heart transplant recipients with recurrent rejection. J Heart Lung Transplant 11: 1283-1289.

11. Zachary AA, Sholander JT, Houp JA, Leffell MS (2009) Using real data for a virtual crossmatch. Hum Immunol 70: 574-579. [Crossref]

12. Stewart S, Winters GL, Fishbein MC, Tazelaar HD, Kobashigawa J, et al. (2005) Revision of the 1990 working formulation for the standardization of nomenclature in the diagnosis of heart rejection. J Heart Lung Transplant 24: 1710-1720. [Crossref]

13. Philogene MC, Zhou S, Lonze BE, Bagnasco S, Alasfar S, et al. (2018) Pre-transplant Screening for Non-HLA Antibodies: Who should be Tested? Hum Immunol 79: 195202. [Crossref]

14. Pena JR, Fitzpatrick D, Saidman SL (2013) Complement-dependent cytotoxicity crossmatch. Methods Mol Biol 1034: 257-283.

15. Hetrick SJ, Schillinger KP, Zachary AA, Jackson AM (2011) Impact of pronase on flow cytometric crossmatch outcome. Hum Immunol 72: 330-336. [Crossref]

16. Philogene MC, Bagnasco S, Kraus ES, Montgomery RA, Dragun D, et al. AntiAngiotensin II Type 1 Receptor and Anti-Endothelial Cell Antibodies: A CrossSectional Analysis of Pathological Findings in Allograft Biopsies. Transplantation.

17. Dragun D, Muller DN, Brasen JH, Fritsche L, Nieminen-Kelha M, et al. (2005) Angiotensin II type 1-receptor activating antibodies in renal-allograft rejection. $N$ Engl J Med 352: 558-569.

18. Dechend R, Homuth V, Wallukat G, Kreuzer J, Park JK, et al. (2000) AT (1) receptor agonistic antibodies from preeclamptic patients cause vascular cells to express tissue factor. Circulation 101: 2382-2387. 
Randhawa PK (2018) Management of heart transplant recipients with hemodynamically significant clinical rejection in the presence of antibodies against angiotensin II type 1 receptor: A retrospective study

19. Dragun D (2007) Agonistic antibody-triggered stimulation of Angiotensin II type 1 receptor and renal allograft vascular pathology. Nephrol Dial Transplant 22: 1819-1822.

20. Dechend R, Müller DN, Wallukat G, Homuth V, Krause M, et al. (2005) Activating auto-antibodies against the AT1 receptor in preeclampsia. Autoimmun Rev 4: 61-65. [Crossref]

21. Navalkar S, Parthasarathy S, Santanam N, Khan BV (2001) Irbesartan, an angiotensin type 1 receptor inhibitor, regulates markers of inflammation in patients with premature atherosclerosis. J Am Coll Cardiol 37: 440-444.

22. Unal H, Karnik SS (2014) Constitutive activity in the angiotensin II type 1 receptor: discovery and applications. Adv Pharmacol 70: 155-174. [Crossref]

23. Takezako T, Unal H, Karnik SS, Node K (2017) Current topics in angiotensin II type 1 receptor research: Focus on inverse agonism, receptor dimerization and biased agonism. Pharmacol Res 123: 40-50.

24. Takezako T, Unal H, Karnik SS, Node K (2015) Structure-Function Basis of Attenuated Inverse Agonism of Angiotensin II Type 1 Receptor Blockers for ActiveState Angiotensin II Type 1 Receptor. Mol Pharmacol 88: 488-501.

25. Zhang S, Zheng R, Yang L, Zhang X, Zuo L, et al. (2013) Angiotensin type 1 receptor autoantibody from preeclamptic patients induces human fetoplacental vasoconstriction. J Cell Physiol 228: 142-148.
26. Carroll RP, Riceman M, Hope CM, Zeng A, Deayton S, et al. (2016) Angiotensin II type-1 receptor antibody (AT1Rab) associated humoral rejection and the effect of peri operative plasma exchange and candesartan. Hum Immunol 77: 1154-1158. [Crossref]

27. Urban M, Slavcev A, Gazdic T, Ivak P, Besik J, et al. (2016) The impact of angiotensin II type 1 receptor antibodies on post-heart transplantation outcome in Heart Mate II bridged recipients. Interact Cardiovasc Thorac Surg 22: 292-297.

28. John R, Lietz K, Schuster M, Naka Y, Rao V, et al. (2003) Immunologic sensitization in recipients of left ventricular assist devices. J Thorac Cardiovasc Surg 125: 578-591.

29. Itescu S, John R (2003) Interactions between the recipient immune system and the left ventricular assist device surface: immunological and clinical implications. Ann Thorac Surg 75: S58-S65.

30. Philogene MC, Jackson AM (2016) Non-HLA antibodies in transplantation: when do they matter? Curr Opin Organ Transplant 21: 427-432. [Crossref]

31. Wiwattanathum P, Ingsathit A, Thammanichanond D, Worawichawong S (2018) Successful Treatment of Anti-angiotensin II Type 1 Receptor Antibody-Associated Rejection in Kidney Transplantation: A Case Report. Transplant Proc 50: 877-880.

32. Taniguchi M, Rebellato LM, Cai J, Hopfield J, Briley KP, et al. (2013) Higher risk of kidney graft failure in the presence of anti-angiotensin II type-1 receptor antibodies. $\mathrm{Am}$ $J$ Transplant 13: 2577-2589. [Crossref]

Copyright: $@ 2018$ Randhawa PK. This is an open-access article distributed under the terms of the Creative Commons Attribution License, which permits unrestricted use, distribution, and reproduction in any medium, provided the original author and source are credited. 